\title{
Deadly DAaRTS destroy cancer cells via a tumor microenvironment-mediated trigger
}

\author{
James V. McCann, ${ }^{1}$ Jamie L. Null, ${ }^{2}$ and Andrew C. Dudley ${ }^{2,3}$ \\ 'Department of Cell Biology and Physiology, University of North Carolina at Chapel Hill, Chapel Hill, North Carolina, USA. Department of Microbiology, Immunology, and Cancer Biology, and \\ ${ }^{3}$ Emily Couric Cancer Center, The University of Virginia, Charlottesville, Virginia, USA.
}

\begin{abstract}
Stromal cells within the tumor microenvironment play a supportive role in tumor growth, progression, and treatment resistance; therefore, these nonmalignant cells are potential therapeutic targets. In this issue of the $J C I$, Szot et al. devised a strategy to exploit the cell-surface marker TEM8 (also known as ANTXR1), which is expressed by cancer-associated stromal cells, as a zip code to deliver an antibody-drug conjugate (ADC) linked to the potent cancer-killing drug monomethyl auristatin $\mathrm{E}$ (MMAE). In preclinical tumor and experimental metastasis models of multiple cancer types, TEM8ADC targeted TEM8-expressing cancer-associated stromal cells, which processed and liberated membrane-permeable MMAE and released this drug via the P-glycoprotein (P-gp) drug transporter. Released MMAE killed cancer cells through a bystander mechanism that did minimal damage to the stromal cells themselves. P-gp-expressing tumor cells displayed MMAE resistance, suggesting that $\mathrm{P}$-gp expression status may identify patients who might benefit the most from TEM8-ADC. This strategy, termed DAaRTS (drug activation and release through stroma), represents an elegant example of how selective expression of a cell-surface molecule on cancer-associated stroma can be exploited to facilitate drug delivery and shrink solid tumors.
\end{abstract}

\section{Targeting tumor stroma for anticancer therapy}

The concept that solid tumors are heterogeneous communities of interdependent cell types, rather than homogeneous spheres of malignant cancer cells, has fundamentally changed the way we think about tumor progression and metastasis (1). Moreover, viewing tumors in this way has revolutionized treatment strategies, because nonmalignant cells (i.e., stromal cells such as endothelial cells, pericytes, fibroblasts, and immune cells) within a tumor may be targeted to elicit anticancer activity. For example, antiangiogenic agents are prototypical stroma-targeted drugs and include both RTK inhibitors and antibodies that block VEGF signaling in the tumor endothelium (2). While the effectiveness of anti-VEGF therapies has been limited by alternative angiogenesis pathways, tumor co-option of pre-existing vessels, and other complex mechanisms, this class of drugs has recently been repurposed and used at suboptimal doses to normalize the vasculature and boost the efficacy of immune checkpoint blockade (3-6). This exciting example illustrates how the cancer stroma can be exploited to achieve maximum benefit in combinatorial treatment regimens.

The effectiveness of stroma-targeted therapies depends, in part, on unique properties of the stromal cells themselves. Thus, distinct antigens expressed in the tumor stroma, especially surface antigens, are attractive targets for selective drug delivery to tumors. Almost 20 years ago,

Related Article: p. 2927

Conflict of interest: The authors have declared that no conflict of interest exists

Reference information: / Clin Invest. 2018;128(7):2750-2753. https://doi.org/10.1172/JCI121527.

Brad St. Croix and coworkers provided a pioneering and highly influential publication that reported the first genome-wide analysis of mRNA expression in human tumor-associated endothelial cells (7). From this study, several antigens were uncovered on the tumor vasculature and named tumor endothelial cell markers (TEMs, TEM1-9). Subsequent studies from the same group and others have confirmed the selective expression of various TEMs in the vasculature in mice and humans and across different tumor types (8). However, some TEMs also frequently appear on vascular cells in healing wounds, in regenerative tissues such as the corpus luteum, and in unexpected cell types, including tumor cells, cancer-associated fibroblasts (CAFs), and other mesenchymal-lineage cells (9). The promiscuous expression of TEMs in the vasculature in these different settings was not unexpected, because gene expression patterns in the endothelium are commonly shared during both pathological and physiological angiogenesis (10).

\section{TEM8-targeting antibody drug conjugate}

In this issue, Szot et al. take advantage of the restricted expression of TEM8 in pathological versus physiological angiogenesis (11). Moreover, while TEM8 was also found in CAFs and pericytes, it was absent from stromal cells in normal regenerating and proliferative tissues and organs. TEM8 is a single-pass, cell-surface transmembrane glycoprotein that has been shown to bind collagen types I and VI and aid in cell spreading and migration on collagen $(12,13)$. Tem 8 -knockout mice are viable, without defects in physiological angiogenesis; however, tumor growth is often delayed in these animals compared with WT mice, suggesting that hostderived TEM8 is tumor supportive (14, 15). Accordingly, antibodies raised against the extracellular domain of TEM8 impair tumor growth in multiple human tumor 


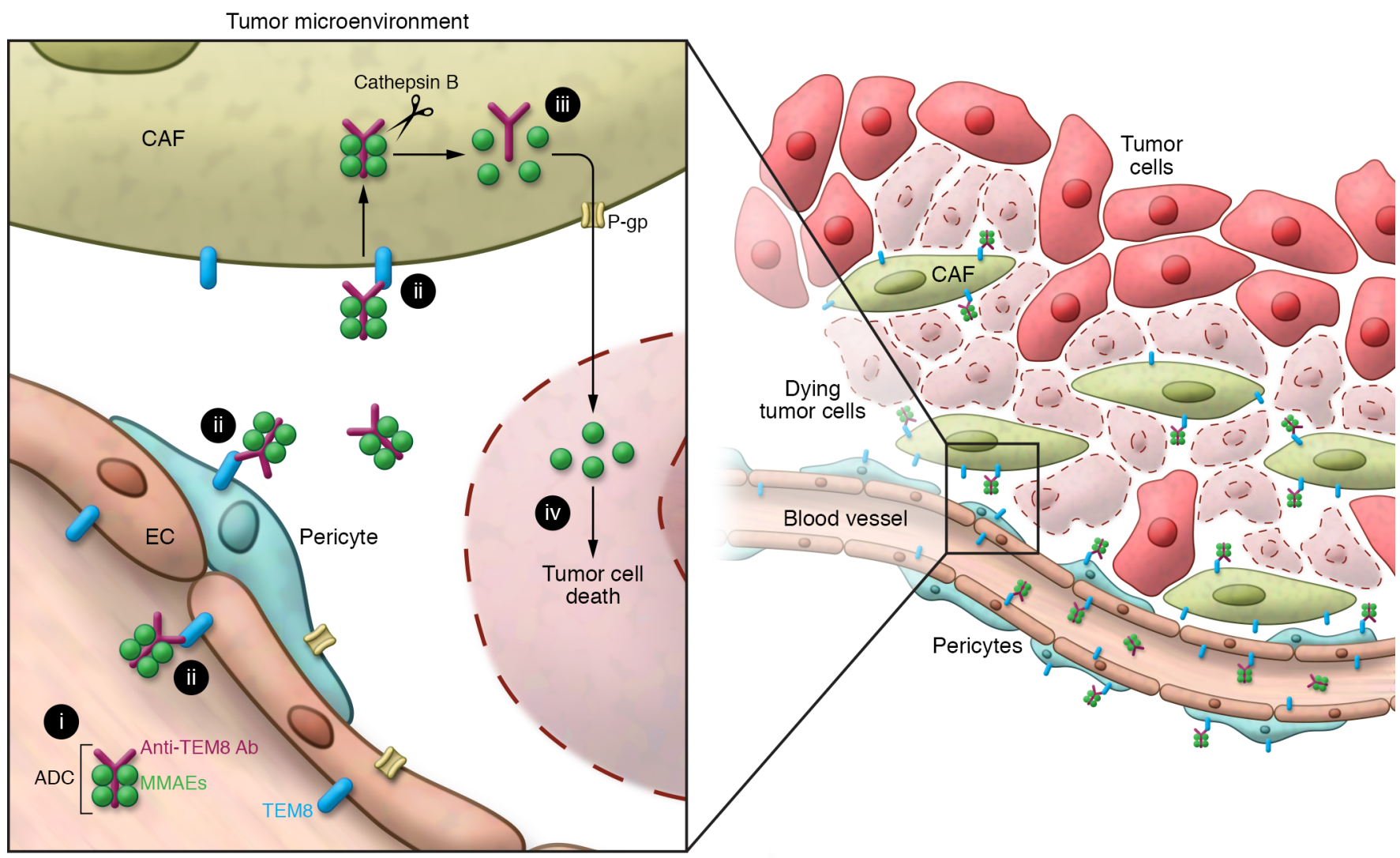

Figure 1. TEM8-ADC kills cancer cells via a bystander mechanism dependent on TEM8-expressing tumor stroma. The efficacy of the TEM8-ADC (i) as an antitumor agent is driven by DAaRTS. TEM8 expression in the tumor stroma, including fibroblasts, pericytes, and endothelial cells, localizes MMAE-linked TEM8-ADC to growing tumors (ii). Upon binding to stromal cells, the ADC is endocytosed and processed by lysosomal proteases such as cathepsin B (iii), thereby liberating cell-permeable MMAE that is exported by the drug transporter P-gp. Once MMAE is evicted from stromal cells, it is bioavailable to the rapidly proliferating cancer cells nearby (iv), where it induces apoptosis (provided these cancer cells do not express abundant P-gp themselves). For simplicity, processing of TEM8-ADC and eviction of free MMAE is only shown in CAFs; however, similar mechanisms may occur in cancer-associated pericytes and endothelial cells that also express TEM8.

xenograft models (15). Thus, while TEM8 appears to provide an advantage to cancer cells because of its auxiliary function(s), selective upregulation of this protein in the stroma also creates a vulnerability for cancer cells that can be exploited by antibody-drug conjugates (ADCs) and other targeted therapies.

ADCs combine the specificity of a $\mathrm{mAb}$ with a covalently linked cytotoxic molecule, thereby allowing for targeted delivery of a lethal payload to cancer cells. There are several critical considerations, including selection of the tumor antigen target, specificity of the antibody against the target, the toxic payload, and the linker connecting the antibody to the payload, that guide successful ADC development (16). Szot and colleagues generated a TEM8-targeting ADC consisting of a TEM8-specific mAb conjugated to monomethyl auristatin E (MMAE, a micro- tubule-disrupting synthetic analog of the natural product dolastatin) by a cathepsin B-cleavable dipeptide linker. In multiple tumor xenografts, including breast, colon, lung, ovarian, and pancreatic cancer cells, TEM8-ADC elicited potent and dose-dependent antitumor activity, whereas equivalent doses of unconjugated TEM8 antibody resulted in only modest reductions in tumor growth. Strikingly, $10 \%$ to $80 \%$ of mice were tumor free following TEM8ADC treatment, depending on the type of cancer. Mice with established orthotopic breast tumors and pancreatic tumor xenografts showed a significant reduction of tumor burden when challenged with TEM8-ADC in combination with doxorubicin or gemcitabine compared with mice given monotherapy; thus, TEM8-ADC is not only effective as a single agent but also augments the efficacy of conventional cytotoxic agents. TEM8-ADC was also shown to have potent metastasis-suppressing activity, as it prolonged survival in models of experimental metastasis to liver and lung. Although further evaluation in models of spontaneous tumor dissemination and genetically engineered mouse models will help confirm the activity of TEM8-ADC in primary tumors and their metastases, taken together, the results of the current study suggest that TEM8-ADC has durable antineoplastic effects at multiple stages of cancer progression.

An important feature of TEM8-ADC is its tolerability in mice. Indeed, toxicological studies demonstrated mild but reversible toxicity in the gastrointestinal tract, an effect that appears dependent on murine carboxylesterase-1C, which cleaves MMAE from the ADC. Surprisingly, CAFs or tumor-associated endothelial cells were highly resistant to the TEM8ADC, even though these cell types express 
abundant TEM8 and internalize MMAE. Szot et al. have elegantly linked this finding to the mode of action of TEM8-ADC itself. Following TEM8-ADC endocytosis by TEM8-expressing stromal cells, MMAE is cleaved by intracellular proteases, such as cathepsin B, into a cell-permeable form that is subsequently exported by the transmembrane ATP-binding cassette drug transporter P-glycoprotein (P-gp). Because TEM8-positive stromal cells divide slowly and express high levels of P-gp compared with surrounding cancer cells, these cells are intrinsically drug resistant, whereas rapidly dividing cancer cells are drug sensitive (Figure 1). Once this bystander mechanism of action of TEM8-ADC was elucidated, Szot and colleagues were able to make predictions about which tumor cells would be TEM8-ADC responders on the basis of P-gp status. In accord with their hypothesis, cancer cells with abundant P-gp were more resistant to TEM8ADC compared with their low P-gp counterparts. Moreover, TEM8-ADC-resistant cells could be sensitized in vitro with P-gp inhibitors. These results suggest that it is possible to predict responsiveness to TEM8-ADC therapy on the basis of P-gp levels in tumor cells.

\section{Perspective}

One of the most impressive and exciting findings in the present study by Szot et al. is the generalizability of TEM8-ADC that is perhaps related to the prevalent TEM8 expression in the tumor stroma at multiple anatomical sites. Using immunohistochemistry, the authors found that TEM8 was expressed in the stroma of approximately $70 \%$ of the tumors studied but was undetectable in almost all corresponding adjacent normal tissues. In 11 tumor models, TEM8-ADC either caused regression or complete inhibition of tumor growth. Even in notoriously hard-to-treat pancreatic cancer, TEM8-ADC diminished tumor growth and extended the length of survival by approximately $175 \%$. Pancreatic cancers are well known to have a dense stromal infiltrate that is thought to act as a barrier against drug delivery, and eliminating this stromal barrier through various pharmacological approaches to improve drug delivery has been attempted (17). Using an opposite approach, Szot and colleagues instead exploit TEM8-express- ing CAFs surrounding pancreatic cancer cells as a means to deliver a lethal warhead. Together, these studies collectively demonstrate some of the creative ways that stroma-targeted therapies are currently being used to kill cancer cells.

An obvious limitation to the present work is the dependency on P-gp for the effectiveness of TEM8-ADC. According to the findings of Szot et al., only patients with low or absent P-gp expression on their cancer cells will respond to TEM8-ADC. Moreover, like other cytotoxicity-based therapies, it is possible that treatment with TEM8-ADC could promote the evolution of more aggressive cancers by inducing P-gp overexpression in tumor cells or selecting for subclones of cancer cells that express abundant P-gp. These P-gpexpressing tumor cells might evict MMAE just as the stromal cells do and potentially escape drug-induced apoptosis. To overcome this possibility, combinations of TEM8-ADCs that kill cancer cells via different mechanisms, or targeting TEM8 itself as an immunotherapeutic strategy using TEM8-directed chimeric antigen receptor $\mathrm{T}$ cells (CAR-T cells) could be used (18). It is possible that the most effective strategy will require combinatorial approaches to attack cancer cells prior to the development of adaptive resistance.

Finally, some questions remain regarding the doses of the TEM8-ADC that are required to obtain maximal efficacy in this study. In particular, the doses used by Szot et al. are considerably higher than those used for most ADCs. Currently, there are over 60 ADC clinical trials, with more than 30 of these trials initiated after 2013, illustrating the excitement and promise surrounding the use of ADCs. Though there were minimal adverse effects in mice treated with high doses of TEM8-ADC, it is unclear whether similar results would be observed in humans. However, judicious combination of the TEM8-ADC with other agents could also help reduce the effective drug doses required in humans. Notably, the St. Croix group recently found that alternative warheads improve dosing and efficacy with a different stromatargeted ADC, suggesting that TEM8ADCs may benefit from further optimization of drug-linker design (19). In sum, the broad expression of TEM8 in the stroma of multiple cancer types and the apparent safety of TEM8-ADC in the preclinical tumor models presented make DAaRTS a versatile and potentially clinically practical approach for treating human patients.

Address correspondence to: Andrew C. Dudley, Department of Microbiology, Immunology, and Cancer Biology, The University of Virginia, Charlottesville, Virginia 22908, USA. Phone: 434.924.7766; Email:acd2g@virginia.edu.

1. Egeblad M, Nakasone ES, Werb Z. Tumors as organs: complex tissues that interface with the entire organism. Dev Cell. 2010;18(6):884-901.

2. Folkman J. Anti-angiogenesis: new concept for therapy of solid tumors. Ann Surg. 1972;175(3):409-416.

3. Carmeliet P, Jain RK. Molecular mechanisms and clinical applications of angiogenesis. Nature. 2011;473(7347):298-307.

4. Schmittnaegel $\mathrm{M}$, et al. Dual angiopoietin-2 and VEGFA inhibition elicits antitumor immunity that is enhanced by PD-1 checkpoint blockade. Sci Transl Med. 2017;9(385):eaak9670.

5. Wu X, et al. VEGF neutralization plus CTLA-4 blockade alters soluble and cellular factors associated with enhancing lymphocyte infiltration and humoral recognition in melanoma. Cancer Immunol Res. 2016;4(10):858-868.

6. Allen E, et al. Combined antiangiogenic and anti-PD-L1 therapy stimulates tumor immunity through HEV formation. Sci Transl Med. 2017;9(385):eaak9679.

7. St Croix B, et al. Genes expressed in human tumor endothelium. Science. 2000;289(5482):1197-1202.

8. Carson-Walter EB, Watkins DN, Nanda A, Vogelstein B, Kinzler KW, St Croix B. Cell surface tumor endothelial markers are conserved in mice and humans. Cancer Res. 2001;61(18):6649-6655.

9. Christian S, et al. Endosialin (Tem1) is a marker of tumor-associated myofibroblasts and tumor vessel-associated mural cells. Am J Pathol. 2008;172(2):486-494.

10. Seaman S, Stevens J, Yang MY, Logsdon D, Graff-Cherry C, St Croix B. Genes that distinguish physiological and pathological angiogenesis. Cancer Cell. 2007;11(6):539-554.

11. Szot C, et al. Tumor stroma-targeted antibody-drug conjugate triggers localized anticancer drug release. J Clin Invest. 2018;128(7):2927-2943.

12. Werner E, Kowalczyk AP, Faundez V. Anthrax toxin receptor 1 /tumor endothelium marker 8 mediates cell spreading by coupling extracellular ligands to the actin cytoskeleton. J Biol Chem. 2006;281(32):23227-23236.

13. Nanda A, et al. TEM8 interacts with the cleaved C5 domain of collagen alpha 3(VI). Cancer Res. 2004;64(3):817-820.

14. Cullen M, et al. Host-derived tumor endothelial marker 8 promotes the growth of melanoma. Cancer Res. 2009;69(15):6021-6026.

15. Chaudhary A, et al. TEM8/ANTXR1 blockade inhibits pathological angiogenesis and poten- 
tiates tumoricidal responses against multiple cancer types. Cancer Cell. 2012;21(2):212-226.

16. Beck A, Goetsch L, Dumontet C, Corvaïa N.

Strategies and challenges for the next generation of antibody-drug conjugates. Nat Rev Drug Discov. 2017;16(5):315-337.
17. Provenzano PP, Cuevas C, Chang AE, Goel VK, Von Hoff DD, Hingorani SR. Enzymatic targeting of the stroma ablates physical barriers to treatment of pancreatic ductal adenocarcinoma. Cancer Cell. 2012;21(3):418-429.

18. Byrd TT, et al. TEM8/ANTXR1-specific CAR
T cells as a targeted therapy for triple-negative breast cancer. Cancer Res. 2018;78(2):489-500.

19. Seaman S, et al. Eradication of tumors through simultaneous ablation of CD276/B7-H3-positive tumor cells and tumor vasculature. Cancer Cell. 2017;31(4):501-515.e8. 